\title{
Correction to: Strain-modulated mechanical, electronic, and thermal transport properties of two-dimensional $\mathrm{PdS}_{2}$ from first-principles investigations
}

\author{
Yang-Shun Lan ${ }^{1}$ - Q Qing Lu ${ }^{1} \cdot$ Cui-E Hu${ }^{2} \cdot$ Xiang-Rong Chen ${ }^{1} \cdot$ Qi-Feng Chen ${ }^{3}$
}

Published online: 16 January 2019

๑) Springer-Verlag GmbH Germany, part of Springer Nature 2019

\section{Correction to: Applied Physics A (2019) 125:33 https://doi.org/10.1007/s00339-018-2311-0}

In the original publication of this article, acknowledgement disclaimer was not published completely.

It should read as follows:

The authors would like to thank the support by the NSAF Joint Fund Jointly setup by the National Natural Science Foundation of China and the Chinese Academy of Engineering Physics under Grant No. U1830101, the Science Challenge Project under Grant No. TZ2016001, and the National Natural Science Foundation of China under Grant No. 11504035.

Furthermore a citation number in Sect. 3.3 was published incorrect.

The sentence: "The transition of indirect to quasi-direct for the $\mathrm{PdS}_{2}$ monolayer is in agreement with the recent result obtained by Miró et al. [23] and Ahmad et al. [23]. The energy-gap difference between our present value and the previous result mainly comes from the calculation..."

should read:

"The transition of indirect to quasi-direct for the PdS2 monolayer is in agreement with the recent result obtained by Miro et al. [23] and Ahmad et al. [40]. The energy-gap difference between our present value and the previous result mainly comes from the calculation..."

We apologize for this error.

The original article has been corrected.

The original article can be found online at https://doi.org/10.1007/ s00339-018-2311-0.

Cui-E Hu

cuiehu@126.com

Xiang-Rong Chen

xrchen@scu.edu.cn

1 Institute of Atomic and Molecular Physics, Sichuan University, Chengdu 610065, China

2 College of Physics and Electronic Engineering, Chongqing Normal University, Chongqing 400047, China

3 National Key Laboratory for Shock Wave and Detonation Physics Research, Institute of Fluid Physics, Chinese Academy of Engineering Physics, Mianyang 621900, China 